\title{
DENSIDAD FONONICA Y POTENCIAL DE DEFORMACION
}

\author{
Luis Salazar De Paz \\ Facultad de Ciencias Físicas, Universidad nacional Mayor de San Marcos \\ Apartado Postal 14-0149, Lima 14. Perú
}

\begin{abstract}
Resumen
En las últimas décadas se ha desarrollado métodos computacionales para la simulación de modelos de sistemas líquidos y amorfos utilizando potenciales de pares para la formulación de la energía total de una determinada sustancia con métodos del espacio real. Ashcroft y Stroud introdujeron pseudo-potenciales sencillos en la formulación teórica de primeros principios, para el cálculo de potenciales de pares; su aplicación se restringió solo a algunos sistemas metálicos simples. El tratamiento con fonones se utiliza en la determinación de una serie de propiedades físicas. En el presente trabajo se ve los sólidos desde el punto de vista de aspectos fonónicos. La intención es contribuir en la descripción de un modelo que coadyuve a determinar las propiedades elásticas de los sólidos por métodos no clásicos.
\end{abstract}

Palabras claves: Fonón, dispersión, Sólidos, potencial de pares, pseudo potencial.

\begin{abstract}
On the latest decades it had developed computer methods for simulation of liquid and amorphous system methods using potential pairs for total energy formulation of a particular substance with real space methods. Ashcroft and Stroud introduced simple pseudo potentials on the theoretical formulation of first beginnings, for pair potential calculation; the application for that had restricted only in a few simple metallic systems. The treatment with phonons is used on the determination of a series of physical properties. On this work it looks the solids from a point of view of the phonon's aspects. The intention is to contribute on the description of a model that assists to determinate the elastic properties of the solids by non-classics methods.
\end{abstract}

Keywords: Phonon, dispersion, solids, pair potential, pseudo potential.

\section{Introducción}

El movimiento de los átomos en la materia pone de manifiesto una serie de fenómenos físicos. En los sólidos, la dinámica de redes se encuentra ligada al movimiento de núcleos y de electrones ligados más próximos de los átomos. Esta dinámica se describe en términos de modos de oscilaciones de la red, que se conoce como fonones [1]. Suele considerarse a los fonones como partículas; aunque realmente no lo son y conviene tratarlas como cuasi-partículas.

Cada modo de vibración se hace equivaler al movimiento de un oscilador armónico. El estado de energía de un modo particular se encuentra determinado por un conjunto de números cuánticos que corresponden a cada modo de oscilación dado. De otro lado, cada modo se identifica por un número de onda $\vec{k}$, o por una frecuencia $\omega$. Un modo de frecuencia $\omega$ puede tener un conjunto de valores de energía discreta, entonces se dice que están presentes fonones de número de onda $\vec{k}$ (o de frecuencia $\omega$ ) donde se manifiestan apariciones y desapariciones denominadas ramas de oscilaciones.

A inicios de la última década del siglo próximopasado, en la Facultad de Ciencias Físicas, se trabajó en la determinación de pseudopotenciales 
aplicables a sistemas con el fin de optimizar los cálculos de potenciales de pares. En ese entonces empecé a desarrollar el tema sobre cálculos de espectros fonónicos y densidad de estados fonónicos en aleaciones metálicas y en semiconductores, usando esos nuevos pseudopotenciales ab initio local [2], era un proyecto de tema abierto a la investigación.

La idea fue usar un pseudopotencial ab initio local para la formulación de un potencial de pares efectivo en aleaciones metálicas $s$ y $p$ para el cálculo de la dispersión fonónica y de la densidad de estados de los fonones del bulk. Esperábamos que un potencial así permitiera tratar especialmente los efectos de largo alcance en la interacción entre los iones efectivos y conectar su efecto en la dispersión fonónica.

El problema fue abordado parcialmente en forma limitada, debido entre otras cosas a que en el cristal se presentan efectos de correlación que no vienen del empaquetamiento geométrico de los otros átomos descritos en los modelos. Las propiedades de simetría de una red infinita se consideran de importancia, ya que el potencial de un electrón individual en una red estacionaria presenta la propiedad de periodicidad $\Phi(\vec{r})=\Phi[\vec{r}-\vec{R}]$. En estas condiciones, haciendo un tratamiento en términos de transformada de Fourier, se puede contar con una función periódica $F(\vec{r})=F[\vec{r}-\vec{R}]$, válida para todo $\vec{R}_{n}$, e integrar sobre toda una determinada celda unidad. Este modelo considera una red cristalina vibrante formada por átomos, cuyas masas se consideran puntuales, acoplados unos a otros por fuerzas tales que la energía potencial total del cristal finito sea en sí finita, y que los desplazamientos atómicos en los puntos de red son realmente muy pequeños. Así, $\vec{u}(n)$ es desplazamiento del átomo $m$ en un punto de la red $\vec{R}_{n}$. En un cristal perfecto, la energía cinética se expresa como $T=\frac{1}{2} M \sum\left[\frac{d u(n)}{d t}\right]^{2}$. Donde $M$ es la masa atómica, idéntica para todos los átomos en el cristal de Bravais monoisotrópico. La energía potencial se expande como

$$
\begin{aligned}
& U=U_{0}+\sum B_{i m} u_{i}(m)+\frac{1}{2} \sum B_{i j m n} u_{i}(m) u_{j}(n) \\
& +\frac{1}{3 !} \sum B_{i j k m n o} u_{i} u(m) u_{j}(m) u_{k}(o)+\ldots
\end{aligned}
$$

Donde $B_{i m}, B_{i j m n}, B_{i j k m n o}$, etc. son constantes de acoplamiento de primer, segundo, tercer orden, etc. En esta ecuación, para la dinámica de redes, la cantidad más importante es la segunda constante de acoplamiento que, evaluada en el equilibrio, toma la forma:

$$
B_{i j m n}=\left\lceil\frac{\partial^{2} U}{\partial u_{i}(m) \partial_{j}(n)}\right\rceil U=0 .
$$

Primero, se construye el hamiltoniano $H$ de una red de átomos interaccionando vía potenciales simples, luego se aplica la aproximación armónica en notación matricial. Los modos normales del cristal explican un poco la simetría que deben obedecer las matrices dinámicas

$$
D\left(\vec{R}-\vec{R}^{\prime}\right)=B_{i j m n} .
$$

Prescindiendo de fuerzas interiores los modos normales explican la simetría general a la que obedecen las matrices dinámicas:

$$
\begin{aligned}
& >D\left(\vec{R}-\vec{R}^{\prime}\right)=D\left(\vec{R}^{\prime}-\vec{R}\right) \\
& >D(\vec{R})=D(-\vec{R}) \\
& >\sum D(\vec{R})=0
\end{aligned}
$$

Se calcula el potencial de pares necesario para la formulación de la energía total del sólido cristalino, mirando el espacio real. Para el cálculo de estos potenciales se requiere de los potenciales atómicos, pero estos presentan una singularidad cuando tienden hacia el centro de la coraza, por este motivo es conveniente reemplazarlos por pseudo-potenciales [3].

Métodos como A.P.W. y R.A.P, dan buenos resultados en este caso, pero introducen una fuerte dependencia de los parámetros del potencial de mufin-tin en la caracterización de los estados. Esto se presenta por la necesidad de incluir muchos orbitales atómicos de diferente momento atómico buscando obtener una buena convergencia de los autovectores $\varepsilon(\vec{k})$ y por el 
enorme grado de complejidad en el cálculo. Por esto se conviene en reemplazarlos por pseudopotenciales $a b$ initio local a fin de eliminar la mencionada singularidad.

La solución de la ecuación de movimiento es equivalente a encontrar una transformación de coordenadas que reducen $\mathrm{T}, \mathrm{U}$ y $\mathrm{H}$ a una suma de cuadrados con términos cruzados. Si T y U son definidas positivas, entonces $\mathrm{T}$ y $\mathrm{U}$ son simultáneamente diagonalizadas.

Posteriormente se encuentra la relación $M \omega_{s}^{2}(k) \varepsilon(\vec{k})=D(\vec{k}) \varepsilon(\vec{k}), \quad$ de cuyas tres soluciones por cada uno de los $\mathrm{N}$ valores permitidos de $\vec{k}$ dan $3 \mathrm{~N}$ modos normales.

Ahora, considerando que la celda se encuentra en la primera zona de Brillouin y a partir de la condición de estabilidad, se determina para la matriz dinámica $D(\vec{k})$, tres autovectores reales cumplen con las condiciones de ortogonalidad y de clausura $\delta_{s}(\vec{k}) \cdot \delta_{s}(\vec{k})=\delta_{s s}, \mathrm{~S}, \mathrm{~S}^{\prime}=1,2,3$. Las frecuencias son $\omega_{s}(\vec{k})=\sqrt{\frac{\lambda_{s}(\vec{k})}{M}}$, donde los valores de $\vec{k}$ están limitados por las condiciones de contorno de Born-Von Karman. La formulación de estos modelos evidentemente no es nada sencilla, es necesario simular sistemas atómicos auto consistentes.

En un cristal anisotrópico los vectores de polarización no tienen la necesidad de estar relacionados con la dirección de polarización a menos que $\vec{k}$ sea invariante bajo ciertas transformaciones de simetría del cristal. El efecto principal de introducir una base poliatómica es producir una rama óptica.

Analizando los resultados, se encuentra que son extrapolaciones del caso considerado. Los modos de vibración acústicos, de longitud de onda larga, están relacionadas con las propiedades elásticas macroscópicas de los sólidos. Caracterizado y calculado el potencial de pares en cristales sencillos se sigue con el cálculo de la dispersión fonónica y la densidad de estados de los fonones de la coraza [2].

\section{Planteamiento del estudio}

El mayor conocimientos de los materiales a utilizarse, nos permite realizar simulaciones teóricas. Tomando como base el modelo expuesto se trata de integrarlo al caso de semiconductores a fin de construir un modelo que describa dispositivos fotovoltaicos. Se abordará en el modelo la interacción electrónfonón [4]; el desplazamiento del borde de la banda de conducción de los cristales y la variación de la masa efectiva del electrón para terminar con un método de acoplamiento intermedio en la teoría de interacción electrónfonón.

\section{Descripción del tema}

\subsection{Modelo de potencial de deformación}

Idealmente, en un cristal, si los iones estuvieran fijos en sus posiciones se podría hablar de una periodicidad perfecta del campo de potencial [5]. Pero, lo cierto es que los iones están en continua agitación térmica debido a la energía interna; este movimiento relativo de los iones alrededor de sus posiciones de equilibrio altera la periodicidad del sistema [6]; bien se podría decir que se altera la variación local del estado electrónico. Y viceversa, también se puede decir que la variación local del estado electrónico ocasiona un cambio local de las fuerzas de interacción entre los átomos y por consiguiente una deformación local de la red y la excitación de las oscilaciones átomos-fonones [5], [7]. Esta interacción se manifiesta incluso a temperatura de cero absoluto. Al aumentar la temperatura aumenta la magnitud de respuesta de la interacción electrón-fonón [8] en las siguientes formas:

- En el cristal se acompañan el movimiento de electrones con el campo de deformación.

- Los electrones cambian de estado de movimiento emitiendo o absorbiendo fonones.

- La interacción electrón - fonón eventualmente conduce a una superconductividad en el material (solo en algunos casos).

En particular en los cristales covalentes (no iónicos), semiconductores tipo $\mathrm{Ge}$ y tipo $\mathrm{Si}$, la interacción electrón-fonón se puede describir 
mediante potenciales de deformación [9], estos potenciales debido a la variación de distancia entre los átomos de la red, caracteriza los estados energéticos de los electrones. Las excitaciones de onda larga desempeñan un papel importante ya que en estos casos el cristal se podría considerar como un continuo [2]. La variación relativa del volumen se puede expresar directamente por medio de los vectores desplazamiento $u(\vec{r})$.

En los cristales cúbicos, cerca de la zona de Brillouin en el espacio $\vec{k}$, las superficies isoenergéticas tienen forma esférica. En la aproximación de masa efectiva, con un cristal ideal, la ley de dispersiónresponde a la relación $E(\vec{k})=\frac{n k^{2}}{2 m^{*}}$. Se encuentra que la región de mayor densidad y por ende de mayor polarizabilidad actúa como un pozo de potencial sobre un electrón de conducción. Se nota que la atracción del pozo sobre el electrón hace que este disminuya su energía deformando al cristal tanto en la posición del electrón como en sus vecindades; interpretamos esto como que la interacción electrón-fonón ocasiona una variación de la ley de dispersión $E\left(\vec{k}, \xi_{v \mu}\right)-E(\vec{k})=W$.

Si la deformación no fuera homogénea, entonces el tensor de deformación $\xi(\vec{r})=\xi(\vec{r})$ dependería de la coordenada $\vec{r}$. En la aproximación adiabática, $W=W_{d}(\vec{r})$ toma el nombre de potencial de deformación quien en cristales isótropos determina el operador de energía de interacción electrón-oscilación acústica de onda larga. Las oscilaciones de la red se pueden descomponer en longitudinales y transversales; se encuentra que las transversales no aportan al potencial de deformación. Introduciendo el vector de desplazamiento $u(\vec{r})$ de un átomo en la posición $\vec{n}$ en la representación de números de ocupación de los estados monoelectrónicos en la banda de conducción [10] con relación al potencial de deformación, e introduciendo los operadores de función de onda $\psi(\vec{r})$, que contienen los operadores escalera de Fermi $\left(W_{d}(\vec{r}), \psi(\vec{r})\right)$, se construye el operador $\mathrm{H}_{\text {int }}$ hamiltoniano de interacción electrón-fonón acústico longitudinal en la representación de los números de ocupación quien define los procesos de dispersión normal

$$
\begin{aligned}
H_{\mathrm{int}} & \equiv \int \psi^{+}(\vec{r}) W_{d}(\vec{r}) \psi(\vec{r}) d^{3} r \\
& =\frac{1}{\sqrt{N}} \sum F_{a}(q) a_{\vec{k}}^{+} a_{\vec{k}}\left(b_{q a}-b_{-q, a}^{+}\right)
\end{aligned}
$$

En esta ecuación cada sumando caracteriza los procesos de emisión y absorción de un fonón por un electrón [2]. En esta expresión se ignora el operador $\mathrm{H}_{\text {int }}^{(u)}$ que describe los procesos de "transferencia" que considera la estructura discreta del cristal. Se sabe que con el operador $\mathrm{H}_{\mathrm{int}}^{(u)}$ incluso los fonones de onda larga pueden dispersar electrones con ángulos muy grandes. A temperaturas suficientemente bajas solo están excitados los fonones de onda larga; aquí los procesos de transferencia carecen de importancia ("se congelan"). Pero, al elevarse la temperatura los procesos de transferencia pueden dar un buen aporte a la resistividad. Los fonones virtuales (deformaciones de red) acompañan a electrones lentos en el cristal covalente. El movimiento lento del electrón en la banda de conducción del cristal covalente va acompañado de una deformación local del cristal [2].

En el lenguaje de la teoría de perturbaciones esto es la descripción del proceso de emisión y posterior absorción de "fonones" virtuales por parte del electrón. Es evidente que esto no es un proceso real de emisión y absorción de fonones, ya que un electrón lento no tiene energía suficiente como para formar fonones reales que requiere de energía $\hbar \Omega_{\vec{q}}$, sino que es el producto solamente del método matemático del modelo utilizado.

Como se sabe, la interacción electrón-fonón ocasiona una variación en la ley de dispersión; hay entonces necesidad de hacer una renormalización de los electrones lentos y la emisión de electrones. Si la energía del electrón fuera pequeña, $\vec{k}<Q$, la variación en la ley de dispersión se redujera solo a un desplazamiento de bandas de la energía $E(\vec{k})$ respecto a su posición, si es que no se tuviera en cuenta la 
interacción con los fonones y al aumento de la masa efectiva del electrón. Así, el estado del cristal con una determinada energía y un vector de onda vienen a ser una combinación compleja de estados monoelectrónicos [8] con fonones virtuales.

Al electrón en este estado a veces se le llama "electrón revestido". El vector de onda de un electrón "desnudo" no es una integral de movimiento, mientras que el estado de un electrón "revestido" con vector de onda que se conserva viene a ser la superposición del vector de onda del electrón y de los vectores de onda del fonón virtual. Para ver esto, eliminamos la energía del electrón "revestido" mediante la segunda aproximación de la teoría de perturbaciones; a causa de la interacción electrón-fonón el fondo de la banda de conducción desciende y la masa efectiva aumenta [11] (solo en el fondo de la banda de conducción).

En algunos semiconductores (y en metales) la energía de los electrones es mucho mayor que la energía de los fonones $\hbar \omega_{\bar{q}}$; esto hace pensar que si realizamos una aproximación podremos llegar a tomar únicamente la interacción electrón-fonón elástica, donde se toma $D(\vec{k}, \vec{q}) \approx D_{-}(\vec{k}, \vec{q})$. Al variar $\vec{k}$ varía el flujo de electrones y por ende la corriente eléctrica en el material $\mathrm{Si}$ se considera una temperatura

superior a la de Debye, el producto $v_{q \hbar \omega_{q}}$ se hace igual a la energía térmica de las modas $q$ de las oscilaciones fonónicas. $\mathrm{Si}$ se considera, además, $\omega_{k q}^{+} \approx \omega_{k q}^{-}$, en términos del volumen de una celda unidad $v$ se encuentra la rapidez de variación del valor medio de $\vec{k}$ en su dirección inicial $\frac{k}{\tau_{k}}=-\frac{d k}{d t}$ en términos del tiempo de relajación del impulso $\tau_{k}^{-1}=\frac{m v \sigma^{2} k^{2}}{\pi \hbar^{3} M c_{a}^{2}} \Theta$. Esto nos lleva a interpretar que a altas temperaturas la resistencia depende de la energía del electrón y que la resistencia varía linealmente con la temperatura. A temperaturas bajas se toma $\omega_{k q}^{+}+\omega_{k q}^{-}$con la cual se llega a la relación

$$
\frac{k}{\tau_{k}}=\frac{m v \sigma^{2} k^{2}}{\pi \hbar^{2} M c_{a}}\left\lceil\frac{4}{5} k^{2}+\frac{\Theta^{5}}{4 \hbar^{6} c_{a}^{5}} \int \frac{x^{4} d x}{e^{x}-1}\right] .
$$

Esto último quiere decir que a bajas temperaturas la resistencia es proporcional a la potencia quinta de la temperatura. Estos resultados, basándonos en la teoría de perturbaciones, para masas efectivas de electrones muy grandes dan módulos elásticos muy pequeños, tal que la interacción electrón de conducción-oscilación acústica longitudinal ocasiona una deformación local de la red poco profunda y en este caso el electrón tendría energía no-discreta para un movimiento estacionario, en este caso el electrón tendría que ir acompañado de una cantidad de fonones virtuales (traslación de deformación local) lo cual haría crecer mucho la masa del electrón.

Un cristal así no es real, así que hay necesidad de corregir este modelo. Ahora, si consideramos que la energía de los átomos de la red es mucho menor que la energía cinética de los electrones se podría pensar en la aplicación de una aproximación adiabática. $\mathrm{Si}$ consideramos al movimiento de los átomos como clásico y el de los electrones como mecánico cuántico vemos que es posible aplicar el método variacional. Considerando al cristal como un continuo, ópticamente isótropo, se ve, despreciando las traslaciones, los estados más bajos del electrón.

La interacción electrón-oscilaciones acústicas longitudinales se caracteriza por la energía potencial determinado por el operador de la energía de interacción electrón-oscilaciones acústicas de onda larga en la representación $\vec{r}$ (potencial de deformación), $W(\vec{r})=-\sigma \rho(\vec{r})$, donde $\rho$ es un tensor de deformación y $\vec{r}$ la coordenada del electrón.

Para el orden cero en la aproximación adiabática de las perturbaciones se obtiene la energía $E\{\varphi, \rho\}$ del sistema $\{$ electrón + red $\}$ en términos de la energía cinética del electrón, la masa efectiva $\mathrm{m}^{*}$ del electrón, una constante elástica $\beta$ y de la función de onda $\psi(\vec{r})$ normalizada. Finalmente, fijando $\rho(\vec{r}), \quad \mathrm{y}$ minimizando la funcional respecto a $\psi(\vec{r})$ se llega a la ecuación 


$$
\left[-\frac{\hbar^{2}}{2 m^{*}} \nabla^{2}-\sigma \rho(\vec{r})-E(\rho)\right] \psi(\vec{r})=0 .
$$

Buscamos la solución del estado más bajo de esta ecuación y con $E\{\varphi, \rho\}$ se obtiene la energía potencial adiabática $E\{\rho\}$, la cual minimizándola se llega a la deformación $\rho(\vec{r})$.

\section{Conclusiones}

La absorción de fonones reales es posible a todas velocidades del electrón. La absorción y emisión de fonones cambia el estado de movimiento del electrón. La variación del vector de onda $\vec{k}$ a lo largo de la dirección inicial hace que simultáneamente varíe la magnitud del flujo de electrones y ocasione la resistencia al paso de la corriente por el cristal. Por consiguiente, para calcular la resistencia de un semiconductor (y de un metal) hay que determinar la velocidad con que varía el valor medio del vector $\vec{k}$ a lo largo de su dirección inicial $\frac{k}{\tau_{k}} \equiv \frac{d k}{d t}$.

En algunos semiconductores la energía de los electrones es mucho mayor que la energía de los fonones $\hbar \omega_{\bar{q}}$; esto hace pensar que si se realiza una aproximación se podría llegar a tomar únicamente la interacción electrón-fonón elástica. A altas temperaturas la resistencia depende de la energía del electrón y la resistencia varía linealmente con la temperatura. A bajas temperaturas la resistencia es proporcional a la potencia quinta de la temperatura. Se trata pues de un acoplamiento fuerte de los electrones con la deformación de la red.

El método variacional lleva a una expresión de la energía $E(\mu)$ en términos de un parámetro variacional positivo adimensional y del parámetro de red $a$, dada por la diferencia de las energías cinética y potencial

$$
E(\mu)=\frac{3 \pi \hbar^{2}}{2 m^{*} a^{2}} \mu^{2}-\frac{\sigma^{2}}{2 \beta a^{2}} \mu^{3} .
$$

En el presente trabajo, la aplicación de la aproximación adiabática fue solamente una postulación, ya que se ignoró la traslación del electrón que se da junto con la deformación; esto hará que mediante un análisis minucioso se encuentre algunos defectos, como que el estado de un electrón de radio pequeño no se ajuste a un modelo de cristal continuo entonces habrá necesidad de una nueva corrección.

\section{Referencias}

[1] Kittel, Charles. "Introduction to Solid State Physics". Jhon Wiley \& Inc. Toronto. p. 157 197. (1971)

[2] Salazar De Paz, Luis, et al.. "Cálculo de dispersión y densidad fonónica en aleaciones metálicas usando potencial de pares". theorema UNMSM Año 2. $\mathrm{N}^{\circ}$ 3. p 66. (1993).

[3] Yin, M.T. - MarvinL. Cohen. "Ab initio calculation of the phonon dispersion relation: Aplication to Si”. Physical Review B Vol 25, $\mathrm{N}^{\circ}$ 6. p. 4317 - 4320. (1982).

[4] Davidov, A. S. "Teoría del sólido". Editorial MIR. Moscu. p, 234 - 288, (1981).

[5] McCurdy, A. K. "Phonon conduction in elastically anisotropic cubic crystals". Physical Review B Vol 26, No 12. p. 6971 - 6986. (1982).

[6] Barden , Jhon - Pines, David. "ElectronPhonon Interaction in metals". Physical Review Vol. 99, p. 1140. (1955).

[7] I. Sham, L. J. - Ziman, J. M. "The ElectronPhonon Interaction". Solid State Physics Vol. 15, p. 221. (1955).

[8] Zabel, L - Magerl, A. - Ruish, J. J. "Phonon in $\mathrm{LiC}_{6}$ ". Physical Review B. Vol. 27, No 6. p. 3930 - 3933. (1983).

[9] Zhongwen LI - Shiwu Gao. "Band-theory calculation oh image state on a metal surface". Physical Review B. Vol. 50, N 20. (Novembe 1994-II).

[10] Madhu Menon - Gerald B. Arnold. "Model Calculations of local electrón and phonon densities of status in bimetallic superlattices". Physical Review B Vol. 27, No 9. p. 5508 5518. (1983).

[11] Radkowsky, A. "Temperature dependance of electronenergy levels in solids". Physic Review, No 73. p. 749. (1948). 